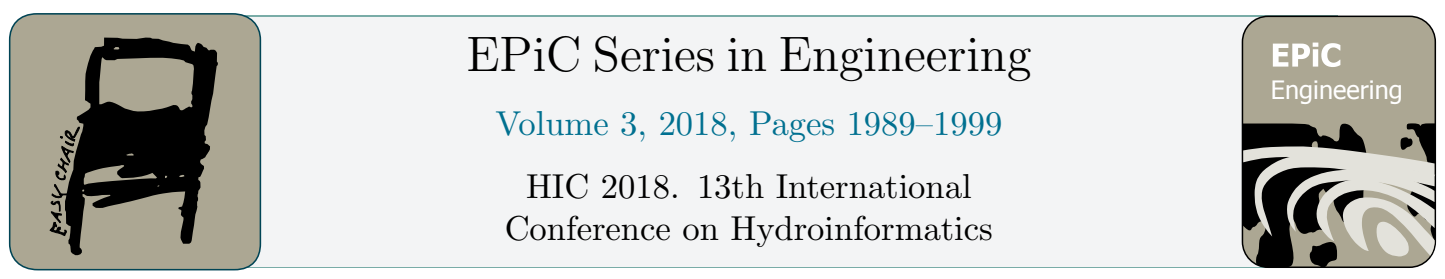

\title{
On migrating to advanced model predictive control strategies at the Moselle River
}

\author{
Franz Simons ${ }^{1}$, Julia Kasper', Kai-Uwe Amann², Eckhard Arnold², \\ Oliver Sawodny ${ }^{2}$, Carsten Thorenz ${ }^{1}$ \\ ${ }^{1}$ Federal Waterways Engineering and Research Institute, \\ Kussmaulstraße 17, 76187 Karlsruhe, Germany \\ 2 Institute for System Dynamics, University of Stuttgart, \\ Waldburgstraße 17/19, 70563 Stuttgart, Germany \\ Corresponding author:franz.simons@baw.de
}

\begin{abstract}
Along the German and Luxembourgian part of the Moselle River, eleven distributed local water level and discharge controllers ensure safe navigation by guaranteeing a water level within a specified tolerance and by reducing variations in the river discharge. The current control scheme is based on gain scheduled PI control with a feed forward disturbance compensation element. Both were parametrized using a 1D SaintVenant model. Due to advancements in control strategies and processing power, the current scheme will be upgraded by adding a model predictive feed forward component (MPFFC) which improves local control and links the isolated local controllers to coordinate their efforts. The authors want to report on this process from an operator's point of view and share their insights from the ongoing testing procedure prior to actual service. A prototype implementation was deployed on the target hardware and linked to the data acquisition system to verify real time operation. The logged results are then verified using a simulation model.
\end{abstract}

Keywords: Model predictive control; waterway operation; water level and discharge control; automatization

\section{Introduction}

The German system of federal inland waterways has a total length of $7350 \mathrm{~km}$, of which $41 \%$ are impounded rivers. Water level control at these impoundments is required to track a desired water level within a specified tolerance to ensure the safety of navigation, to guarantee flood water discharge, to reduce variations in the 
On Migrating to Advanced Model Predictive Control Strategies at the Moselle River F. Simons et al.

river discharge and to allow using the full hydraulic energy potential. It is a demanding task to fulfill all these concurrent requirements. In the past, water level control was successfully done manually. Accordingly, the control quality was mainly dependent on the knowledge and experience of the operator. A challenge in the ongoing automatization of the impoundments is the integration of this expert knowledge into control algorithms, which can be applied efficiently to different impoundments. The aim is to standardize the management of impoundments with an easy to comprehend water level control, which ensures robust and safe operation of the waterways.

The study area is the Moselle River, which rises in France and flows into the River Rhine in the city of Koblenz, Germany. A $394 \mathrm{~km}$ long part from the city NeuvesMaisons in France to the mouth in Germany is impounded and used for navigation and energy production. The water level is therefore controlled by 25 barrages: 13 in France, 2 in German-Luxembourgian condominium and 10 in Germany (Figure 1).

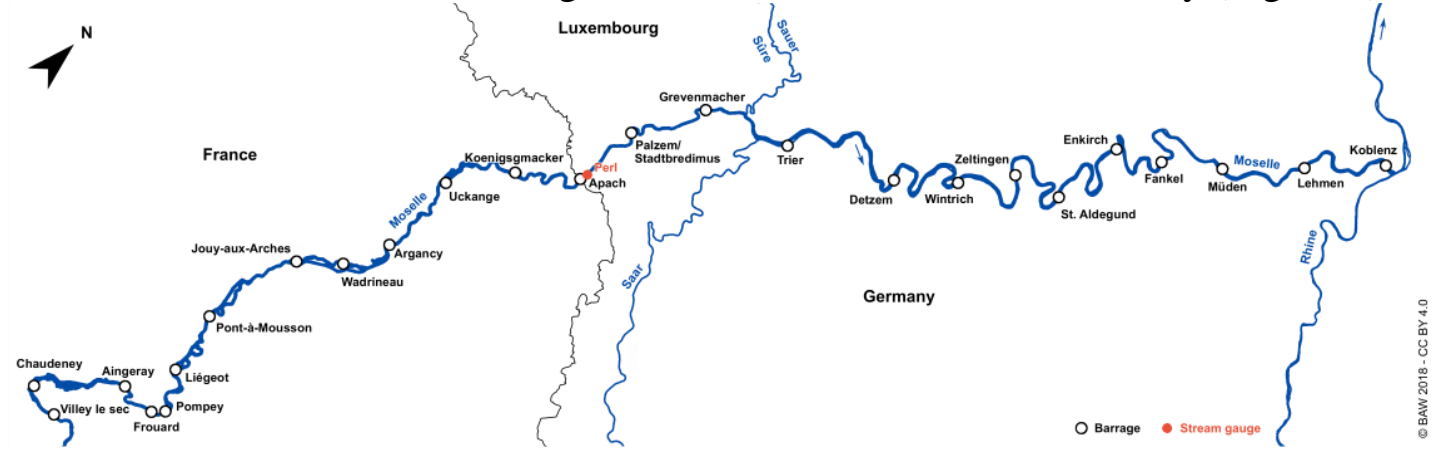

Figure 1 Locations of the 25 barrages at Moselle River

In the years 2001 to 2008 , the water level control was automated at eleven barrages in Luxembourg and Germany, respectively. Since 2008 all controllers are in productive operation without any major problems. The current control solution is a local water level and discharge control as described e.g. in $[1,2,3]$. The water level at the downstream weir is controlled by a PI control loop with added feed forward compensation of upstream discharge variations. Due to distinctive discharge dependent behavior, a single parametrization is not sufficient. Therefore, parameters are obtained at multiple discharge and water level set points based on simulations using a 1D Saint-Venant model of the impoundment and are scheduled during online operation based on the current operating conditions [4,5]. This local control strategy has the disadvantage of only using locally available information, which are the upstream inflow and the downstream water level of the impoundment. Information such as the discharge of the neighboring river impoundments is not considered in the determination of the control strategy. This is in contrast to manual control, where the operators intuitively use information from upstream river sections to anticipate local 
water level deviations and control the downstream discharge accordingly. They also communicate verbally to coordinate control action for significant changes in overall discharge and desired water levels. The water level is lowered with rising discharge to minimize flood risk in the upper part of the impoundment. The transition of one water level set point to another has to be coordinated in the chain of impoundments to avoid the amplification of discharge variations. The aim is therefore to upgrade the current control strategy to allow for coordinated water level and discharge management in a chain of barrages and improve the control performance and navigational safety.

In a joint research project with the University of Stuttgart, a model predictive feed forward control (MPFFC) strategy is currently being developed and deployed to achieve these goals [6]. The chosen approach is based on a control scheme presented in [7], where a full model predictive controller based on linearized 1D Saint-Venant equations is proposed. Simulations using numerical models of the Moselle River reaches in question have so far shown promising results.

In our contribution, we want to report on the migration process from an operator's point of view. First, we will explain the development approach. Then, the state-ofthe-art control strategy is described. In the fourth section the MPFFC strategy developed by the University of Stuttgart is presented and the advantages of the new strategy will be pointed out. We will then give insights in the prototype implementation at a real impoundment which is currently deployed and show results from the ongoing test. Also, the difficulties of obtaining reliable discharge data will be pointed out. The paper will end with a conclusion on the presented topic.

\section{Model-based design}

A model-based design approach is used for the development, analysis, simulation and deployment of the control strategies. The software packages MATLAB ${ }^{\circledR} \&$ Simulink $^{\circledR}$ are used as development platform [5]. The complex control system is represented by a structured model which represents the framework for the development, analysis and communication throughout the design process. Each part of the model represents a component of the controlled system and is based on a mathematical-physical relationship to describe the behavior of the component. The modular structure allows the individual test and reuse of each component. The developed control strategy can be directly ported to the embedded system by automatic code generation for the specified target platform. From an operator's point of view, the model-based design process facilitates communication and collaboration between different fields of expertise and allows a standardized implementation of the developed algorithms due to the direct deployment to the target platform. 
On Migrating to Advanced Model Predictive Control Strategies at the Moselle River F. Simons et al.

\subsection{D Saint-Venant model}

In the model-based design process, a nonlinear 1D Saint-Venant model of each impoundment is used to represent the controlled system. In addition the model will be used as a reference in the parametrization and linearization processes as it will be shown later. The used software is an in-house development of the Federal Waterways Engineering and Research Institute which solves the transient 1D SaintVenant equations with an implicit Preissmann scheme. The software has an interface to the Simulink ${ }^{\circledR}$ software which allows direct coupling into simulations of the control system. The discretized 1D Saint-Venant model is build up from crosssections at a distance of 50 to $100 \mathrm{~m}$ in flow direction and was calibrated by variation of the Manning friction coefficients.

\section{Water level and discharge control}

Figure 2 shows the structure of the currently deployed water level and discharge control. It shows a typical river reach with an up- and downstream barrage. The discharge of the impoundment is split into the discharge over the weir and the discharge through the power plant. The inflow consists of the measurable discharge of the upstream river reach and possible unknown lateral inflows. The controlled water level is measured at a stream gauge situated directly upstream of the barrage. In the water level control loop the measured water level is compared to the desired water level.

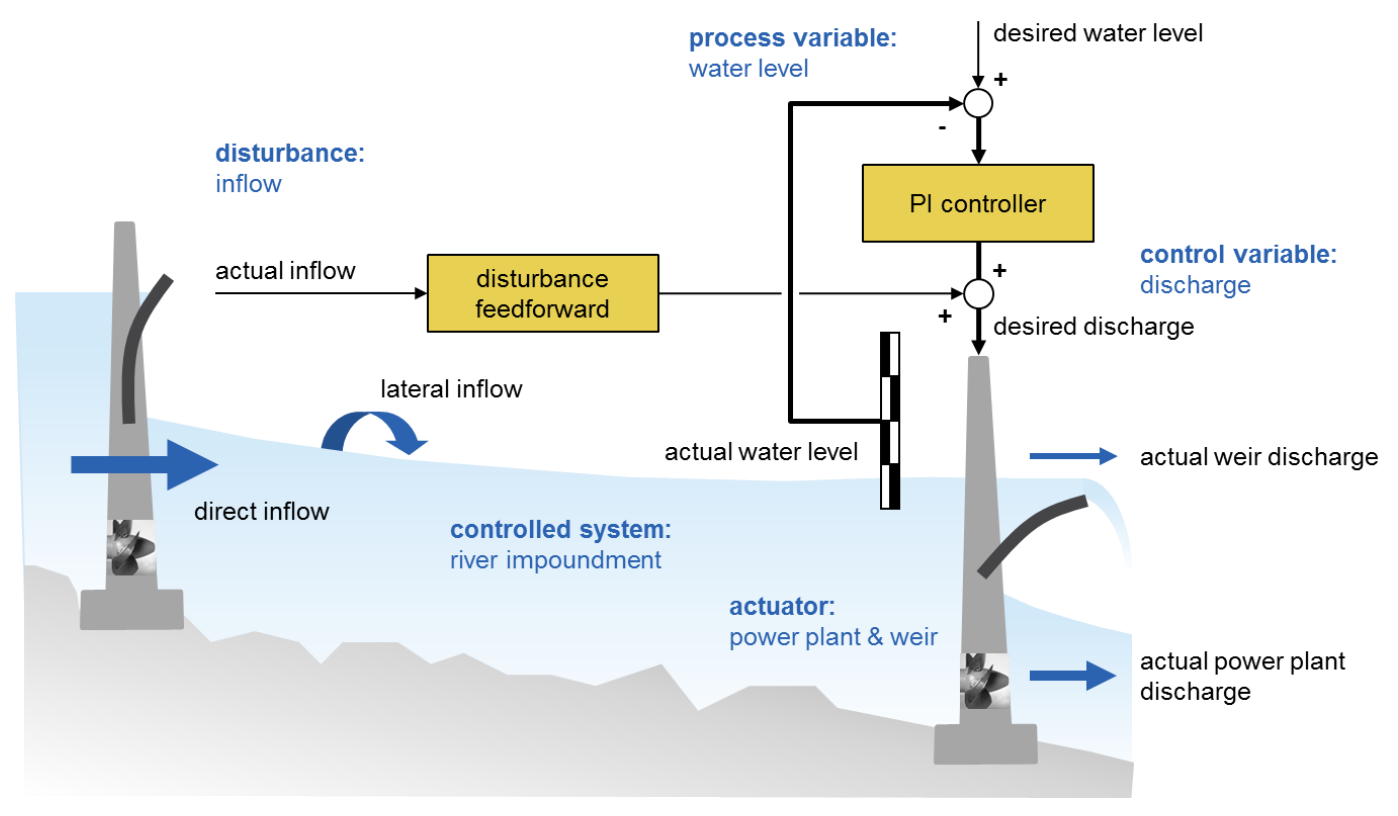

Figure 2 Local PI control loop with disturbance feed forward for river impoundment 
The deviation is used as the input for the PI controller, which computes a desired discharge. The desired discharge is then realized by the power plant and the weir, with respect to their mechanical/physical limitations. This results in a closed control loop. In control engineering terms, the water level in the impoundment is the process variable, the discharge is the control variable and the barrage and power plant are the actuators. The river impoundment is the controlled system. Due to the nonlinear behavior of the controlled system, the discharge-dependent proportional gain and integral time constant are found by brute-force optimization during the PI parameterization. To reduce discharge variations close to the water level set point, the control parameters are tuned to more conservative values in this region, effectively attenuating the controller's actions.

The described water level control allows automated tracking of the desired water level independently of variations in the impoundment inflow. Due to the wave propagation time, there will always be a delay between the variation of the inflow and the reaction at the stream gauge. Especially at low discharge conditions, where the propagation time is large, this delay can cause problems in the control quality. These problems can be reduced by a disturbance feed forward control. A desired discharge is predicted from the current inflow (the disturbance of the system). The disturbance feed forward design is a simplified interpretation of the diffusive wave propagation processes. The translation, i.e. temporal shift of an inflow disturbance, is described by an inflow-dependent time delay. The dispersion of the inflow disturbance due to retention is described by a linear first order low pass filter. The filter coefficient is found by fitting to the results of the nonlinear 1D Saint-Venant model and considering the probability distribution of the river discharge [4].

\section{Model predictive feed forward control}

As shown in Figure 3, the feed forward part of the original water level and discharge control is replaced by the MPFFC. To work properly, the proposed control scheme requires a prediction of the desired water level and of the future upstream inflow. In case of rivers, the short term prediction of the inflow disturbances is feasible as long as there are distant upstream discharge measurements available and the river geometry and management strategy is known. The time horizon used for the predictions is three hours and the discretization time step is one minute. Using a linearized Saint-Venant model, an optimal discharge trajectory is found, which compensates the predicted inflow and fulfills an optimized desired water level trajectory. To cover the full discharge range of the Moselle River, the used model is switched at runtime, where each model has a different nominal discharge [6]. To allow for a smooth transition between two models, the results of the models are interpolated in an overlap region. 
On Migrating to Advanced Model Predictive Control Strategies at the Moselle River F. Simons et al.

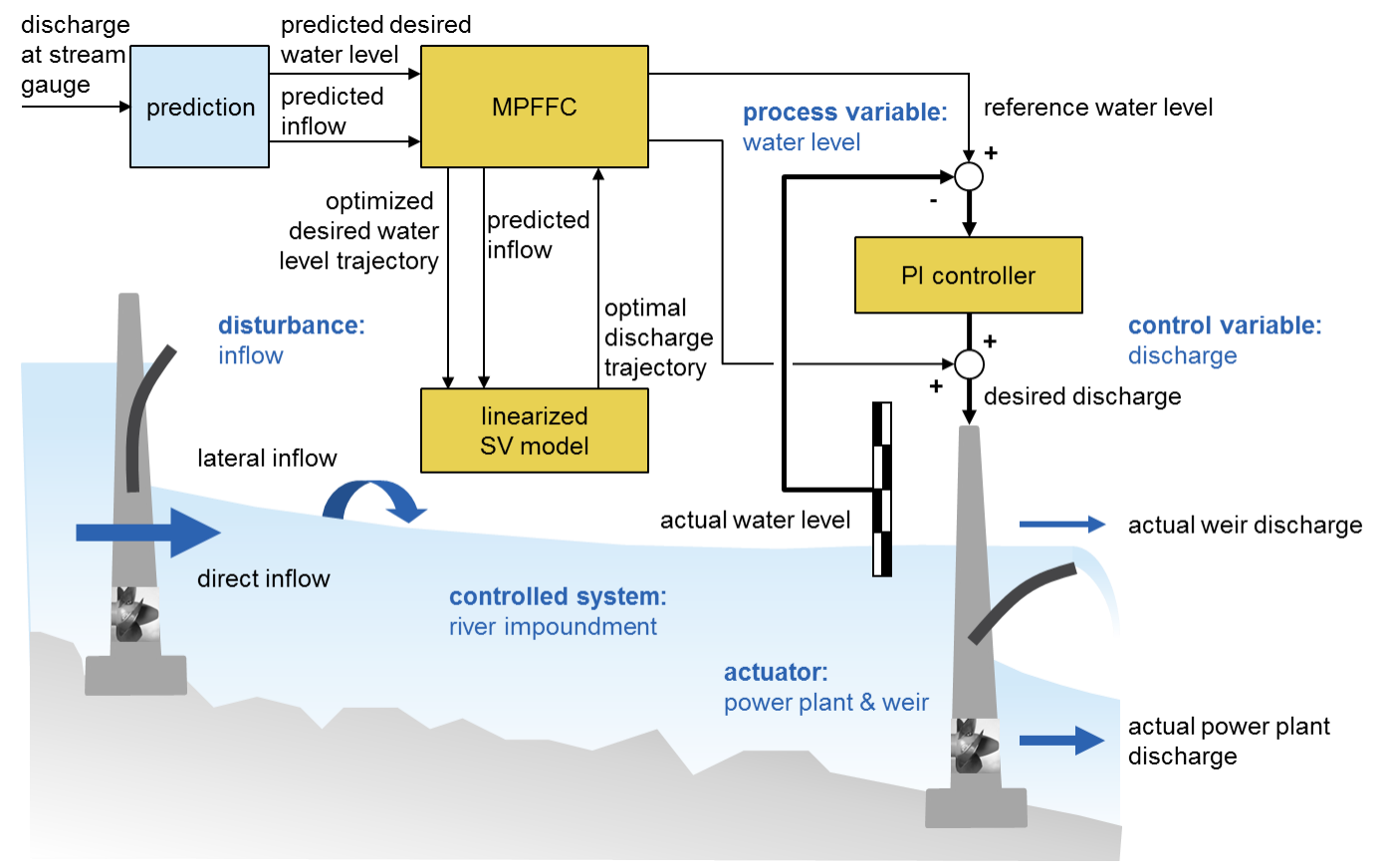

Figure 3 MPFFC with PI control loop

At each time step, the first value of the three hours long water level and discharge trajectories is used as reference water level for the PI controller and is added as a feed forward component to the desired discharge, respectively. In the next time step the optimization process will be started again using the newest inflow predictions.

In contrast to the control system presented in Section 3, the PI controller in the MPFFC control system is not needed for discharge attenuation, but can be optimized for water level tracking. The reduction of discharge variations by utilization of the available water level tolerance will be considered as a quality criterion in the online optimization process of the MPFFC. This explicit consideration of the requirements allows a much more efficient and intuitive parametrization of the control system.

In a chain of barrages, the optimized discharge trajectory of an upstream controller can now be used as an estimate of the future inflow for the next downstream impoundment. Doing so, the new control strategy can be deployed progressively starting from the most upstream impoundment. This is a crucial feature if the long realization phase in a modernization project of up to a decade is considered. In addition, the control software is designed as modular as possible. MPFFC and PI controller can be parametrized and implemented separately. In particular, this is important, as it allows a simplified integration into the existing control system. 
On Migrating to Advanced Model Predictive Control Strategies at the Moselle River F. Simons et al.

Before updating the whole system, the MPFFC can be implemented and tested using the existing PI controller.

\section{Prototype implementation and results}

A prototype implementation of the described control system was deployed on the control hardware on site. The Detzem impoundment was the selected for the field test, as its barrage will be the first one which will be upgraded. The inflow into the impoundment is measured at the upstream barrage Trier (cp. Figure 1). The prototype is computing real-time values for the optimal discharge and reference water level. These values are logged but not yet used in the actual water level control. As seen in Figure 3, there is no feedback of the actual water level and outflow of the impoundment into the MPFFC. It is therefore reasonable to test the MPFFC detached from the PI controller. Using the model of the control system, it is possible to simulate the behavior of the system in retrospect.

In the prototype implementation the inflow is predicted from real-time discharge measurements at the stream gauge Perl, which is located downstream of the barrage Apach at the French border (cp. Figure 1). A prediction for the inflow time series in Detzem is computed by forward simulation of a simplified model of the reach from Perl to Detzem. In the prototype implementation the model consists of a secondorder low pass filter and a delay. The parameters are adjusted at runtime by comparing the first minutes of the prediction time series and the actual inflow measured in the past at Detzem. Figure 4 shows a comparison of the predicted inflow and the actual inflow to the Detzem impoundment over seven days. Here, the predictions three hours and one hour in advance are shown. A good agreement of the phases can be recognized. In addition, it can be seen how the prediction improves over time, due to the runtime adjustment of the parameters. In the operation of the prototype, we observed recurrent problems with the discharge measurements from the stream gauge, like complete failure or unrealistic low discharge. It shows that reliable measurements are crucial for the operation of the MPFFC. In addition, the current prediction has to be improved. Larger tributary rivers as the rivers Saar and Sauer are not considered yet. Therefore, improving the prediction method is currently still under investigation for the planned deployment.

Based on the prediction presented before, Figure 5 shows the logged output of the MPFFC. In addition the results from the simulation of the control system using the nonlinear 1D Saint-Venant model as controlled system are shown. To complete the control system, the PI controller from the currently implemented control scheme is used. The behaviour of the actuators is considered by a minimum step size for the movement of the weir and a delay between two movements. 
On Migrating to Advanced Model Predictive Control Strategies at the Moselle River F. Simons et al.

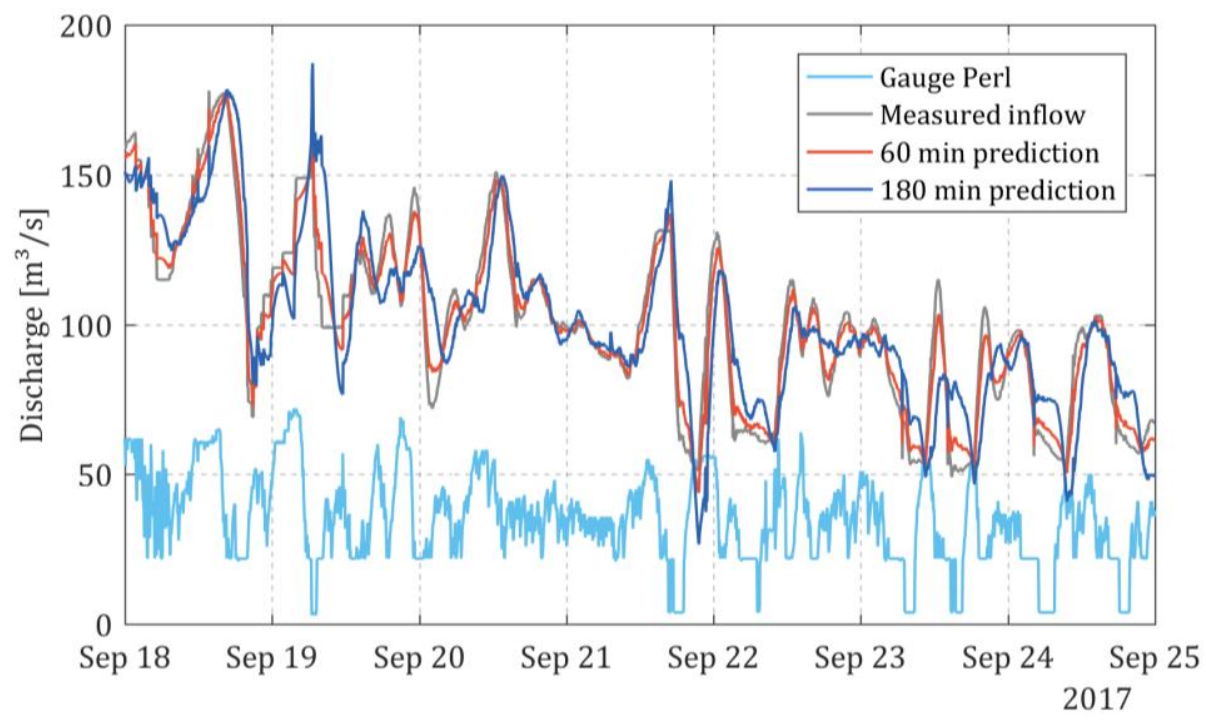

Figure 4 Comparison of the predictions based on real-time measurements at stream gauge Perl and measured inflow to Detzem impoundment. The predictions three hours and one hour in advance are shown.
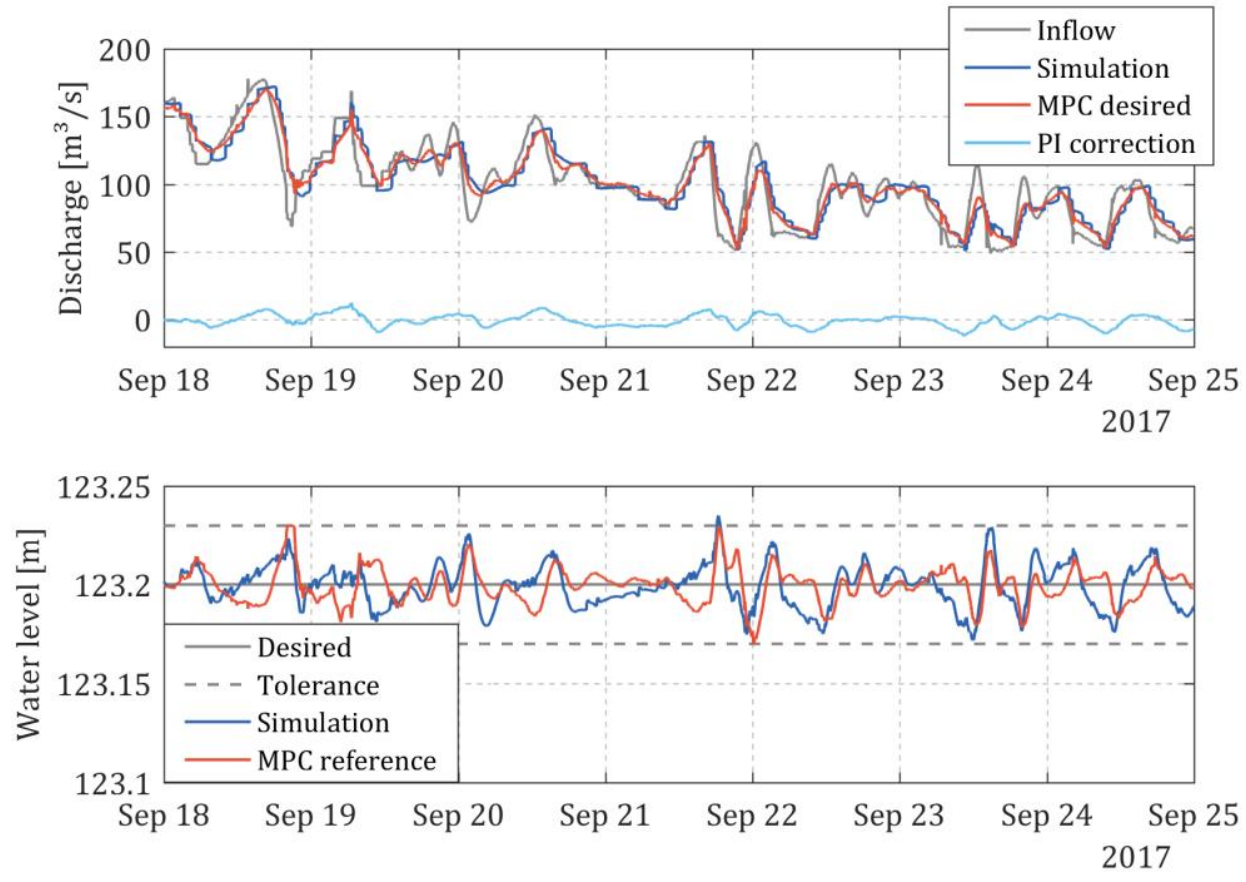

Figure 5 Comparison of water level and discharge computed by the MPFFC prototype (red) and the simulated MPFFC control system using the nonlinear 1D Saint-Venant model as controlled system and a PI controller (blue). In addition the PI correction is shown (light blue). 
On Migrating to Advanced Model Predictive Control Strategies at the Moselle River F. Simons et al.

A very good agreement of the simulated discharge and the one desired by the MPFFC can be recognized. There is an overall good agreement of the water level, too. This indicates that the linearized Saint-Venant models are a good approximation to the real, nonlinear 1D Saint-Venant model. To further support this observation, the discharge correction computed by the PI controller is shown in the upper graph in Figure 5, too. Its magnitude is below $10 \%$ of the discharge computed by the MPFFC. As the model inaccuracies have to be compensated by the PI controller, this points out an overall good agreement of the linearized Saint-Venant models with the real, nonlinear Saint-Venant model, too.

Next, the simulation results are compared to the actual values realized by the currently implemented control scheme described in Section 3. Figure 6 shows the measured discharge and water level at the barrage Detzem and the simulated discharge and water level of the control system. It can be recognized, that the MPFFC anticipates the discharge variations and reduces them by using the available tolerance for the water level.
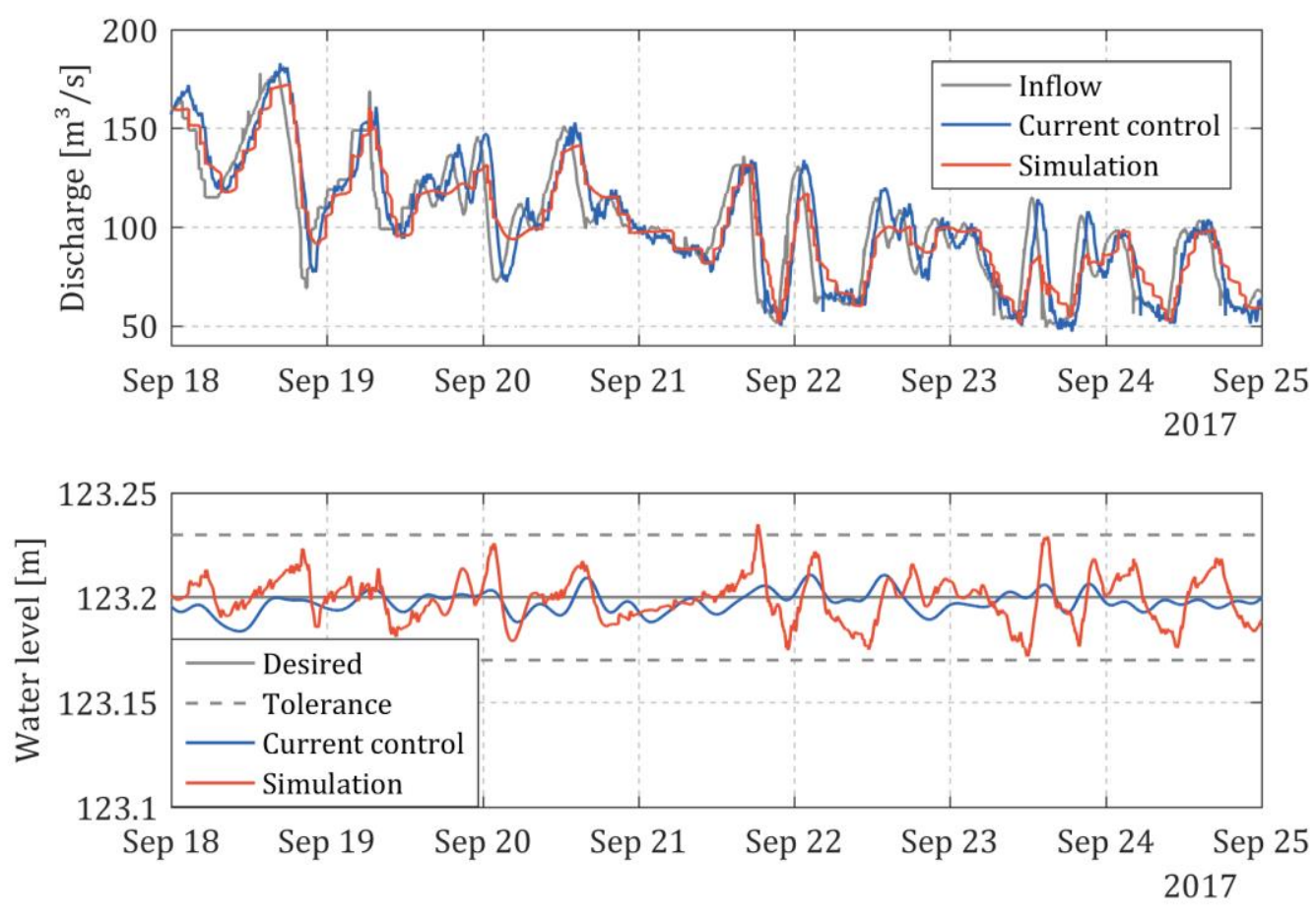

Figure 6 Comparison of discharge and water level realized by the currently implemented control system (blue) and the simulated MPFFC control system using the nonlinear

1D Saint-Venant model as controlled system and a PI controller (red) 
On Migrating to Advanced Model Predictive Control Strategies at the Moselle River F. Simons et al.

\section{Conclusions and outlook}

The current control scheme at the German-Luxembourgian barrages will be upgraded by adding a MPFFC component which improves local control and links the isolated local controllers to coordinate their efforts. Using simulations based on 1D Saint-Venant models, the authors showed in [8] that the developed MPFFC strategy is suitable for water level and discharge control in a chain of barrages. In [6] it was shown that the use of multiple linearized models is necessary to cover the full discharge range. A prototype implementation of the developed control system was deployed on the control hardware on site. The logged data from the field test at the Detzem impoundment allowed investigating the suitability of the presented concepts. The field test showed the importance of reliable data for inflow prediction. Further investigations for the final deployment are necessary. The logged output of the MPFFC shows good agreement of the simplified linearized and the nonlinear SaintVenant models. Using this output in simulations of the whole control system, a reduction of discharge variations could be observed without harming the water level tolerance. This shows the strength of the MPFFC strategy to consider strict requirements in automated water level and discharge control.

In the next step, the MPFFC strategy will be set in productive operation at the Detzem impoundment. After successful operation the new strategy will be deployed progressively at the barrages downstream. Then, the full potential of the MPFFC strategy to effectively attenuate discharge variation in a chain of barrages can be shown.

\section{Reference}

[1] S. Theobald, A. Celan, and F. Nestmann, "Optimization of river system operation for multi-purpose objectives," in HydroVision, Portland, Oregon, 2002.

[2] U. Theobald and S. Theobald, "Central water resources management in a cascade of hydropower plants," in International Conference on Networking, Sensing and Control, Delft, The Netherlands, 2011, pp. 104-108.

[3] M. Gebhardt and S. Wohlfart, "The automatic control of a chain of barrages - from preliminary studies to system start-up of the automated Moselle barrages," $B A W$ Mitteilungen, no. 96, pp. 135-144, 2012.

[4] P. Schmitt-Heiderich and F. Belzner, "Method for the Parameterisation of the PI Contollers for Automated Discharge and Water Level Control," BAW Mitteilungen, no. 96, pp. 123-133, 2012.

[5] M. Gebhardt, P. Schmitt-Heiderich, and S. Wohlfart. „Automated operation of chains of barrages-Development of controller algorithms with the use of model-based design." In Proc. River Flow 2010, Karlsruhe, 2010, pp. 1399-1406

[6] K.-U. Amann, E. Arnold, and O. Sawodny, "Online real-time Scheduled Model Predictive Feedforward Control for impounded River Reaches applied to the Moselle 
On Migrating to Advanced Model Predictive Control Strategies at the Moselle River F. Simons et al.

River," in Proc. of the IEEE Int. Conf. on Automation Science and Engineering, Forth Worth, USA, 2016, pp. 1276-1281.

[7] G. Hug-Glanzmann, M. von Siebenthal, T. Geyer, G. Papafotiou, and M. Morari, "Supervisory water level control for cascaded river power plants," in Hydropower Conference 05, Stavanger, Norway, 2005.

[8] K.-U. Amann, E. Arnold, and O. Sawodny, "Comparison of model based feed-forward control strategies for impounded rivers," in European Control Conference (ECC), 2014, June 2014, pp. 1361-1366. 